and previous findings suggest a novel immune signaling mechanism by which a peptide fragment of high molecular-weight kininogen (HK-D5) acts as an accelerant of both innate and adaptive immune cell chemotaxis in multiple immune contexts. This has broad implications regarding a mechanism of immune-mediated inflammation in a variety of disease states, which might be amenable to the targeting this pathway for therapeutic intent.

3013

Combined Annulus Fibrosus and Nucleus Pulposus Repair Prevents Degeneration in the Ovine Lumbar Spine

Stephen Sloan ${ }^{1}$, Christoph Wipplinger ${ }^{1}$, Sirtac Kirnaz ${ }^{1}$,

Rodrigo Navarro-Ramirez ${ }^{1}$, Antonella Schivinato ${ }^{2}$, Roger Härtl ${ }^{1}$ and

Lawrence Bonassar ${ }^{1}$

${ }^{1}$ Cornell University and ${ }^{2}$ Fidia Farmaceutici

OBJECTIVES/SPECIFIC AIMS: The objective of this study is to assess the efficacy of combined AF and NP repairs to prevent degenerative changes and restore native disc morphology in an in vivo large animal model. We hypothesize that combined repairs will prevent disc degeneration following injury to a greater extent than the individual repairs after 6 weeks in vivo, as demonstrated through disc height measurements and disc morphology. METHODS/STUDY POPULATION: A total of 8 skeletally mature female Finn sheep were used in this study. Following a previously described method, IVDs from L1 to L6 of the lumbar spine were exposed using a lateral access, extraperitoneal approach5. IVDs were randomized into 5 treatment groups: 1) intact discs, 2) discs injured via a $3 \mathrm{~cm} \mathrm{x} 1 \mathrm{~cm}$ box annulotomy and partial nucleotomy, 3) injury followed by a high density collagen (HDC) AF patch, 4) injury followed by injection of a modified hyaluronic acid (HA) into the NP, and 5) injury followed by both the HDC AF patch and HA NP injection. The HDC treatment was $15 \mathrm{mg} / \mathrm{mL}$ type-I collagen mixed with $0.06 \mathrm{mM}$ riboflavin, injected at the defect site and crosslinked in situ with blue light. The NP injection was HA modified with $\mathrm{C} 16$ side chains to increase the viscosity of the hydrogel (HYADD $4^{\circledR}$ )6. At 6 weeks post-operatively, sheep were sacrificed and had 3T magnetic resonance images (MRI) taken of their lumbar spine. Disc height analysis and Pfirrmann grading were performed on each segment using MR images. Additionally, quantitative MRI analyses were performed using a custom MATLAB algorithm that segments NP from the surrounding tissue and directly measures the NP volume. ANOVA with Tukey's HSD was used to determine statistical significance between groups for disc height and quantitative MRI analyses, and the Kruskal-Wallis test with Mann-Whitney tests was used to statistically analyze Pfirrmann Grades. All animal use followed approved IACUC protocol. RESULTS/ANTICIPATED RESULTS: As shown in axial MR images (Figure 1A), intact discs had hyperintense NP with a distinct border to the AF. The discs receiving injury with no treatment had hypointense NP with no distinct border between the AF and NP. Individual and combined treatment with the HA NP injection and HDC AF patch appeared to preserve the hyperintense NP signal and AF/NP border. Intact control discs were not degenerated and had an average Pfirrmann grade of 1 (Figure 1B), while injured, untreated discs had significant degeneration with an average Pfirrmann grade of 3. Discs receiving the HA NP injection and collagen AF patch individually showed fewer signs of degeneration than the injured alone, and the combined treatment resulted in the least amount of degeneration with Pfirrmann grades not significantly different than the intact controls.
Disc height index confirmed the trends seen in the Pfirrmann grades (Figure 1C), where injured discs lost $20 \%$ of the intact disc height, the individual NP and AF repairs restored 5-10\% of intact disc height, and the combined repairs preserved $90 \%$ of the intact disc height. The NP voxel count of all treatment groups were similar to the intact controls (Figure 1D). DISCUSSION/SIGNIFICANCE OF IMPACT: The objective of this study was to determine how combined AF and NP can prevent degenerative changes to the disc in a large animal in vivo model. Pfirrmann grading and disc height index results show that the greatest preservation of disc morphology was seen with combined AF and NP repairs, while the individual strategies prevented degenerative changes better than injury with no treatment. It appears the HA NP injection restores lost water content to the disc following injury, and the AF collagen patch plays a role in maintaining the NP repair within the disc. This is the first study to our knowledge to attempt combined AF and NP repairs in an in vivo large animal model. Combining NP and AF repairs leads to significantly improved outcomes following disc injury, which warrants the translation of combined repairs into the clinic to improve patient outcomes with degenerative disc disease involving $\mathrm{NP}$ and AF.

3528

\section{Common Mechanisms Underlying Epilepsy and Tauopathy}

Ryan Adam Cloyd ${ }^{1}$, Joe Abisambra ${ }^{2}$ and Bret Smith ${ }^{1}$

${ }^{1}$ University of Kentucky Center for Clinical and Translational Science and ${ }^{2}$ University of Florida

OBJECTIVES/SPECIFIC AIMS: Neurologic disorders are among the most significant health challenges facing society today. Although different neurologic disorders are often thought to be distinct from one another, evidence suggests similar processes may contribute to pathology in different diseases. Previous studies suggest that common disease mechanisms contribute to the development of epilepsy and tauopathy. The purpose of this study is to better characterize this relationship and explore potential therapeutic avenues to slow disease progress. METHODS/STUDY POPULATION: This study uses the pilocarpine-induced status epilepticus model of temporal lobe epilepsy to explore the effect of severe seizures on tau pathology. Brains were collected from mice at 6 or 24 hours after induced status epilepticus. Homogenates were analyzed via Western blot to look for changes in tau phosphorylation or activity of two major regulators of tau phosphorylation, GSK3 $\beta$ and PP2A. These data show that changes in tau phosphorylation dynamics occur at a much earlier time point after status epilepticus than has previously been described. RESULTS/ANTICIPATED RESULTS: GSK3 $\beta$ activity increased within 6 hours and remained elevated by 24 hours. PP2A activity initially decreased but returned to normal by 24 hours. These data show that changes in tau phosphorylation dynamics occur at a much earlier time point after status epilepticus than has previously been described. DISCUSSION/SIGNIFICANCE OF IMPACT: The current project supports previous observations that seizures promote tau phosphorylation in vivo, but suggests that changes begin much earlier than previously thought. Further work is needed to understand how post-seizure changes in tau phosphorylation develop over longer periods of time. Additionally, future work will characterize the effect of tauopathy on electrical activity in vivo and in vivo. 